\title{
Correction to: Fully fuzzy multi-item two-stage fixed charge four- dimensional transportation problems with flexible constraints
}

\author{
Sudeshna Devnath $^{1}$ (D) $\cdot$ Pravash Kumar Giri $^{2} \cdot$ Seema Sarkar Mondal ${ }^{1} \cdot$ Manoranjan Maiti $^{3}$
}

Published online: 27 November 2021

(C) Springer Nature Switzerland AG 2021

\section{Correction to: Granular Computing}

https://doi.org/10.1007/s41066-021-00295-x

In the original version of this article, the given and family name of Seema Sarkar Mondal was incorrectly structured. The name was displayed correctly in all versions at the time of publication.

The original article has been corrected.

The original article can be found online at https:// doi.org/10.1007/s41066-021-00295-x.

Sudeshna Devnath

sd.16ma1102@phd.nitdgp.ac.in

Pravash Kumar Giri

pragiri_su2010@yahoo.com

Seema Sarkar Mondal

seema.sarkar@maths.nitdgp.ac.in

Manoranjan Maiti

mmaiti2005@yahoo.co.in

1 Department of Mathematics, National Institute of Technology Durgapur, Durgapur 713209, India

2 Department of Mathematics, Government General Degree College, Dantan-II, Kashmuli 721445, India

3 Department of Applied Mathematics with Oceanology and Computer Programming, Vidyasagar University, Midnapore 721102, India 